\title{
Prediction of modal parameters of linear time-varying systems
}

\author{
S. Marchesiello*, A. Bellino and L. Garibaldi \\ Politecnico di Torino, Corso Duca degli Abruzzi 24, 10129 Torino, Italy
}

\begin{abstract}
Many engineering structures, such as cranes, traffic-excited bridges, flexible mechanisms and robotic devices exhibit characteristics that vary with time and are referred to as time-varying or nonstationary. In particular, linear time-varying (LTV) systems have been often dealt with on a case-by-case basis. Many concepts and analytic methods of linear time-invariant (LTI) systems cannot be applied to LTV systems, as for example the conventional definition of modal parameters. In fact, LTV systems violate one of the assumptions of the conventional modal analysis, which is stationarity.

Subspace-based identification methods, proposed in the 1970s, have been attracting much attention due to their affinity to the modern control theory, which is based on the state space model. These methods are now successfully applied to many industrial cases and may be considered reference methods for identifying LTI systems.

In this paper the use of a subspace-based method for identifying LTV systems is discussed and applied to both numerical and experimental systems. More precisely a modified version of the SSI method, referred to here as ST-SSI (Short Time Stochastic Subspace Identification) is introduced as well as a method for predicting time-varying stochastic systems using the angle variation between the subspaces; the latter is able to predict the system parameter in the "near" future.
\end{abstract}

Keywords: Time-varying, frozen technique, subspace rotation

\section{Introduction}

Modelling and identification of LTV systems are usually treated by two approaches: the ARMA (Auto Regressive Moving Average) methods [1] and the state-space models, which are those preferred by authors to analyse complex systems. Their popularity is also due to the recent increase of interest in subspace-based identification methods for state-space model realizations [2,3]. In [4-6] LTV systems are analyzed with a state-space model with time-varying matrices. Since some time-invariant relations are no more valid, new definitions of the modal parameters can be formulated [5] and some numerical and experimental verifications were proposed [7,8].

A lot of existing algorithms based on the state-space method are founded on the assumption that multiple experiments are conducted on the system with parameters undergoing the same variation, but this is not acceptable for all the LTV systems, so a procedure which uses a set of free or random excitation response data is needed [6].

The idea of prediction of LTV systems is due to Kameyama [9], who tries to predict the future behaviour of a system looking at the rotation of the subspace generated by the columns of the observability matrix at two different time instants. The Kameyama's work is the convergence of three different research backgrounds: the linear algebra, the control theory and the system identification [10].

The predictive approach can be very useful in analyzing systems on which measurements can not be done more frequently. The systems for which the prediction can be applied are called slowly-varying systems [5], i.e. systems whose matrices change slowly with time.

This paper, after a first part regarding the time-variant identification, deals with some mathematical preliminary aspects on the relations between the subspaces generated by the columns of two different matrices. Then the predictive method is described and a numerical and an experimental example are presented in order to validate the algorithms.

\footnotetext{
*Corresponding author. E-mail: stefano.marchesiello@polito.it.
} 


\section{Frozen technique}

The identification technique adopted is based on Van Overschee's work but it is applied to time-variant systems. The main idea is to consider the matrices constant during each time step and, under this assumption, to apply all the results concerning the time-invariant systems. This procedure is often called frozen technique.

The state-space model, for a LTV system, can be written as

$$
\left\{\begin{array}{l}
x(k+1)=A(k) x(k)+B(k) u(k)+w(k) \\
y(k)=C(k) x(k)+D(k) u(k)+v(k)
\end{array}\right.
$$

where $A, B, C$ and $D$ are the system matrices, $x$ is the state vector, $y$ is the output vector, $w$ and $v$ are the state noise and the output noise, respectively.

The whole time period is divided in many windows, in which the system is considered time-invariant. The windows are chosen superimposed and shifted by the sampling period.

The length of the temporal window is indicated by the parameter $L_{f}$, which cannot be chosen arbitrarily, but it must respect the following condition:

$$
L_{f} \geqslant 2 i(l+1)-1
$$

where $l$ is the dimension of the output vector and $i$ is a user defined index, that should be large enough with respect to the maximum order of the system to be identified [2]. For each window the identified matrices are assigned to the central point of that window. It is possible to derive the eigenvalues of the identified state matrix $\hat{A}(t)$, from which the natural frequencies $f_{j}(t)$ are obtained.

\section{Mathematical concepts}

In this section some mathematical aspects are illustrated [9], in order to better understand the predictive method, which will be presented in the next section.

\subsection{Angle between subspaces}

Consider two matrices $A \in \Re^{r \times p}$ and $B \in \Re^{r \times q}$, where $r \geqslant p, q$, with $\operatorname{rank}(A)=a_{d}$ and $\operatorname{rank}(B)=b_{d}$, respectively. The angle between two subspaces spanned by the column vectors of $A$ and $B$ is defined by a set of angles

$$
\left\{\vartheta_{i}, i=1,2, \ldots, m\right\}, \quad m=\min \left(a_{d}, b_{d}\right), \quad 0 \leqslant \vartheta_{i} \leqslant \pi / 2
$$

between the principal vectors $a_{i} \in \operatorname{span}_{\text {col }}\{A\}$ and $b_{j} \in \operatorname{span}_{\text {col }}\{B\}$, where $i, j=1,2, \ldots, m$.

The set of principal vectors and principal angles is called angle between subspaces.

The quickest way to calculate the principal vectors of $\operatorname{span}_{\operatorname{col}}\{A\}$ and $\operatorname{span}_{\operatorname{col}}\{B\}$ is to use the singular value decomposition (SVD), to construct the matrix $Q \in \Re^{r \times r}$.

$$
Q=A\left(A^{T} A\right)^{\dagger} A^{T} B\left(B^{T} B\right)^{\dagger} B^{T}
$$

where the symbol $\dagger$ indicates the pseudo-inverse matrix. Then the SVD is performed:

$$
Q=U S V^{T}
$$

where $U \in \Re^{r \times r}$ and $V \in \Re^{r \times r}$ are orthonormal matrices, $S \in \Re^{r \times r}$ is the matrix containing the singular values $\left\{\sigma_{1}, \ldots, \sigma_{m}\right\}$ of $Q$. The principal vectors of $\operatorname{span}_{\text {col }}\{A\}$ and $\operatorname{span}_{c o l}\{B\}$ are given by $u_{i} \in \Re^{r}$ and $v_{i} \in \Re^{r}$, where $u_{i}$ and $v_{i}$ are the first $m$ column vectors of $U$ and $V$, respectively. The $i$ th principal angle is obtained from the $i$ th singular value with the following expression:

$$
\sigma_{i}=\cos \vartheta_{i} \Rightarrow \vartheta_{i}=\arccos \sigma_{i}, i=1,2, \ldots, m
$$

When $\sigma_{i}=1$, it means that the angle between $u_{i}$ and $v_{i}$ is zero and therefore there is no rotation. 


\subsection{Rotation of the subspace}

From now, the case $p=q$ will be considered, for simplicity and because of greater applicative interest. The column vectors in the $p$-dimensional subspace are

$$
u_{i}=\left[u_{i 1} u_{i 2} \ldots u_{i p}\right]^{T} \in \operatorname{span}_{\text {col }}\{U\} \quad v_{i}=\left[v_{i 1} v_{i 2} \ldots v_{i p}\right]^{T} \in \operatorname{span}_{\text {col }}\{V\}
$$

and they are linked by

$$
v_{i}=R_{\vartheta_{i}} u_{i}
$$

To extract the rotation operator $R_{\vartheta_{i}}$, it is necessary to define the orthonormal base in the rotation plane.

The first two vectors of this base identify the rotation plane in which $u_{i}$ and $v_{i}$ lie:

$$
e_{i 1}=\frac{u_{i}}{\left\|u_{i}\right\|}=u_{i} \quad e_{i 2}=\frac{v_{i}-<v_{i}, e_{i 1}>e_{i 1}}{\left\|v_{i}-<v_{i}, e_{i 1}>e_{i 1}\right\|}
$$

where $<f, g>$ denotes the inner product $f^{T} g$ of $f$ and $g$; $\|f\|$ indicates the Euclidian norm of $f$.

The other vectors of the orthonormal base of $\Re^{r}$ are built such that $e_{i j}^{T} e_{i k}=\delta_{j k}$, for $j, k=1,2, \ldots, p$, where $\delta_{j k}$ indicates the Kronecker operator. The vectors $u_{i}$ and $v_{i}$ can be written as

$$
u_{i}=a_{i 1} e_{i 1}+a_{i 2} e_{i 2}+\ldots+a_{i p} e_{i p}=a_{i 1} e_{i 1}=e_{i 1} \quad v_{i}=b_{i 1} e_{i 1}+b_{i 2} e_{i 2}+\ldots+b_{i p} e_{i p}=b_{i 1} e_{i 1}+b_{i 2} e_{i 2}(10)
$$

where $a_{i j}=<u_{i}, e_{i j}>$ and $b_{i j}=<v_{i}, e_{i j}>, j=1,2, \ldots, p$. It is easy to obtain that $b_{i 1}=\cos \vartheta_{i}$ and $b_{i 2}=\sin \vartheta_{i}$.

Writing the variables $b_{i j}$ in function of the variables $a_{i j}$ and doing some mathematical steps, it is possible to obtain the relation Eq. (8) where

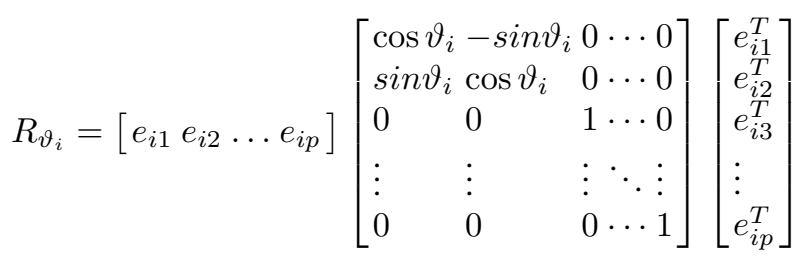

\section{The predictive method}

The predictive method proposed in this section intends to predict the future modal response of the system under study, based on the past and the present behavior. This process can only be applied after having used an estimation method, as discussed in Section 2, because it needs the estimated system matrices. With the dynamic matrix $\hat{A}(k)$ and the output matrix $\hat{C}(k)$, the extended observability matrix at the instant $k$ is calculated:

$$
\hat{\Gamma}_{\alpha}(k) \stackrel{\operatorname{def}}{=}\left[\begin{array}{l}
\hat{C}(k) \\
\hat{C}(k) \hat{A}(k) \\
\hat{C}(k) \hat{A}(k)^{2} \\
\vdots \\
\hat{C}(k) \hat{A}(k)^{\alpha-1}
\end{array}\right]
$$

The symbol ^ means that the term is derived from an estimation procedure. $\hat{\Gamma}_{\alpha}(k) \in \Re^{\alpha l \times n}$, where $n$ is the model order and $\alpha$ is a parameter that can be chosen freely.

By considering three temporal instants $k_{1}, k_{2}$ and $k_{3}$, respectively in the past, in the present and in the future, the proposal of this method is to predict the extended observability matrix at the instant $k_{3}$, once it has been estimated at the instants $k_{1}$ and $k_{2}$. This step can be done since the change of subspace occurs because of the rotation of the column vectors $\hat{\gamma}_{i}(k)(i=1,2, \ldots, n)$ of $\hat{\Gamma}_{\alpha}(k)$. The angle between the subspaces spanned by $\operatorname{span}_{\text {col }}\left\{\hat{\Gamma}_{\alpha}\left(k_{1}\right)\right\}$ and $\operatorname{span}_{c o l}\left\{\hat{\Gamma}_{\alpha}\left(k_{2}\right)\right\}$ is the result of the rotation of each column vector $\hat{\gamma}_{i}(k)$ in the time interval $k_{2}-k_{1}$, in the 
$\alpha l$-dimensional vector space. From (4) and (5), the principal vectors of $\operatorname{span}_{\text {col }}\left\{\hat{\Gamma}_{\alpha}\left(k_{1}\right)\right\}$ and $\operatorname{span}_{\text {col }}\left\{\hat{\Gamma}_{\alpha}\left(k_{2}\right)\right\}$ can be written as

$$
\hat{\Gamma}_{\alpha}\left(k_{1}\right)\left(\hat{\Gamma}_{\alpha}\left(k_{1}\right)^{T} \hat{\Gamma}_{\alpha}\left(k_{1}\right)\right)^{\dagger} \hat{\Gamma}_{\alpha}\left(k_{1}\right)^{T} \hat{\Gamma}_{\alpha}\left(k_{2}\right)\left(\hat{\Gamma}_{\alpha}\left(k_{2}\right)^{T} \hat{\Gamma}_{\alpha}\left(k_{2}\right)\right)^{\dagger} \hat{\Gamma}_{\alpha}\left(k_{2}\right)^{T}=U\left(k_{1}\right) S\left(k_{2} \mid k_{1}\right) V^{T}\left(k_{2}\right)
$$

where

$$
U\left(k_{1}\right)=\left[\hat{\gamma}_{1}\left(k_{1}\right) \hat{\gamma}_{2}\left(k_{1}\right) \ldots \hat{\gamma}_{n}\left(k_{1}\right) \ldots \hat{\gamma}_{\alpha l}\left(k_{1}\right)\right] \quad V\left(k_{1}\right)=\left[\hat{\gamma}_{1}\left(k_{2}\right) \hat{\gamma}_{2}\left(k_{2}\right) \ldots \hat{\gamma}_{n}\left(k_{2}\right) \ldots \hat{\gamma}_{\alpha l}\left(k_{2}\right)\right]
$$

while $S\left(k_{2} \mid k_{1}\right)$ is the diagonal matrix containing the singular values $\hat{\sigma}_{i}\left(k_{2} \mid k_{1}\right)=\cos \hat{\vartheta}_{i}\left(k_{2} \mid k_{1}\right)$.

Let indicate with $\tilde{\gamma}_{i}\left(k_{3} \mid k_{2}\right)$ the predictive estimation of $\hat{\gamma}_{i}\left(k_{3}\right)$ based on the data till to the present time $k_{2}$. Under the hypothesis that the principal vectors rotate always in the same plane, the following relationship holds:

$$
\tilde{\gamma}_{i}\left(k_{3} \mid k_{2}\right)=R_{\hat{\vartheta}_{i}\left(k_{3} \mid k_{2}\right)} \hat{\gamma}_{i}\left(k_{2}\right)
$$

Let define the angular velocity of the angle $\hat{\vartheta}_{i}\left(k_{2} \mid k_{1}\right)$ as

$$
\Delta \hat{\vartheta}_{i}\left(k_{2} \mid k_{1}\right)=\frac{\hat{\vartheta}_{i}\left(k_{2} \mid k_{1}\right)}{k_{2}-k_{1}}
$$

and suppose it constant during all the evolution of the system. In such a way the rotation angle $\hat{\vartheta}_{i}\left(k_{3} \mid k_{2}\right)$ can be predicted with the following formula:

$$
\tilde{\vartheta}_{i}\left(k_{3} \mid k_{2}\right)=\Delta \hat{\vartheta}_{i}\left(k_{2} \mid k_{1}\right)\left(k_{3}-k_{2}\right)
$$

Consequently the prediction $\operatorname{span}_{\text {col }}\left\{\tilde{\Gamma}_{\alpha}\left(k_{3}\right)\right\}$ is given by the principal vectors $\tilde{\gamma}_{i}\left(k_{3} \mid k_{2}\right)$ in the following manner:

$$
\operatorname{span}_{c o l}\left\{\tilde{\Gamma}_{\alpha}\left(k_{3}\right)\right\}=\operatorname{span}_{\text {col }}\left\{\left[\tilde{\gamma}_{1}\left(k_{3} \mid k_{2}\right) \tilde{\gamma}_{2}\left(k_{3} \mid k_{2}\right) \ldots \tilde{\gamma}_{n}\left(k_{3} \mid k_{2}\right)\right]\right\}
$$

$\tilde{\Gamma}_{\alpha}\left(k_{3}\right)$ and $\tilde{\Lambda}_{\alpha}\left(k_{3}\right)=\left[\tilde{\gamma}_{1}\left(k_{3} \mid k_{2}\right) \tilde{\gamma}_{2}\left(k_{3} \mid k_{2}\right) \ldots \tilde{\gamma}_{n}\left(k_{3} \mid k_{2}\right)\right]$ are not the same matrix but they span the same vector space and so they lead to similar matrices.

To extract the modal parameters, it is necessary to obtain the dynamic matrix from $\tilde{\Lambda}_{\alpha}\left(k_{3}\right)$. Let denote with $\tilde{\chi}_{1}\left(k_{3}\right)$ the matrix composed by the first $\alpha-1$ blocks of $\tilde{\Lambda}_{\alpha}\left(k_{3}\right)$ and with $\tilde{\chi}_{2}\left(k_{3}\right)$ the matrix composed by the last $\alpha-1$ blocks of $\tilde{\Lambda}_{\alpha}\left(k_{3}\right)$, then

$$
\tilde{\chi}_{2}\left(k_{3}\right)=\tilde{\chi}_{1}\left(k_{3}\right) \tilde{A}\left(k_{3}\right)
$$

and finally

$$
\tilde{A}\left(k_{3}\right)=\left(\tilde{\chi}_{1}\left(k_{3}\right)\right)^{\dagger} \tilde{\chi}_{2}\left(k_{3}\right)
$$

In the examples considered, the predictive method will be used to predict the system at many instants, defining the instants $k_{2}$ and $k_{3}$ at each step in the following manner:

$$
k_{2}=k_{1}+d \quad k_{3}=k_{2}+p_{r}
$$

The parameters $d$ and $p_{r}$ are called building factor and prediction factor, respectively. The latter must be chosen by respecting the following condition:

$$
p_{r}>\frac{L_{f}}{2}
$$

The relation Eq. (22) is imposed in order to avoid "false prediction", i.e. when the instant $k_{3}$ is included in the window centered in $k_{2}$.

The goodness of the prediction results is linked to two factors: the time-variability of the system (already cited in the introduction) and the choice of $d$ and $p_{r}$. In fact if $d>>p_{r}$ the results will be very good, if $d<<p_{r}$ they will be unacceptable. 


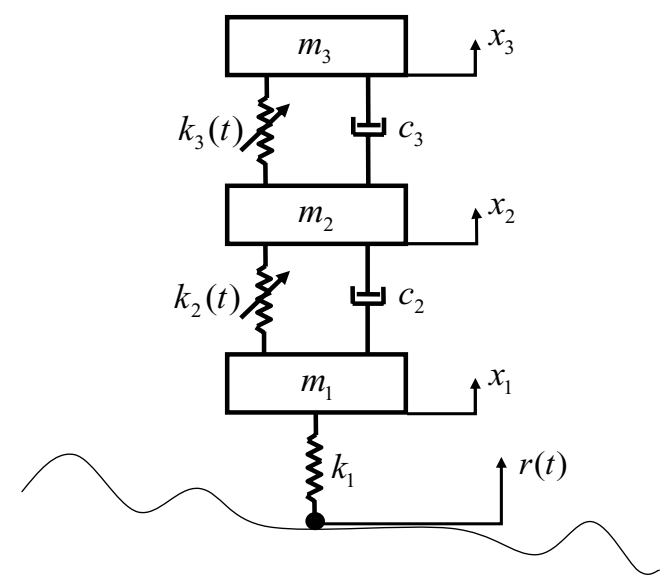

Fig. 1. The mechanical system considered.

\section{Numerical example: model of a scaled truck}

Consider the mechanical system depicted in Fig. 1, consisting of three masses, three springs and two dampers [1]. The system is a model of a reduced scale truck: $m_{1}$ is the mass of the wheel, $m_{2}$ is the body mass and $m_{3}$ is the cabin mass.

The non-stationarity is due to the springs $k_{2}$ and $k_{3}$, whose time-varying law is defined by

$$
k_{i}(t)=k_{i 0}+k_{i 1} \sin \left(\frac{2 \pi t}{p_{i 1}}\right)+k_{i 2} \sin \left(\frac{2 \pi t}{p_{i 2}}\right), \quad i=2,3
$$

where $p_{i 1}$ and $p_{i 2}$ are the characteristic periods of the temporal variation.

The mass matrix, the damping matrix and the stiffness matrix are expressed as follows:

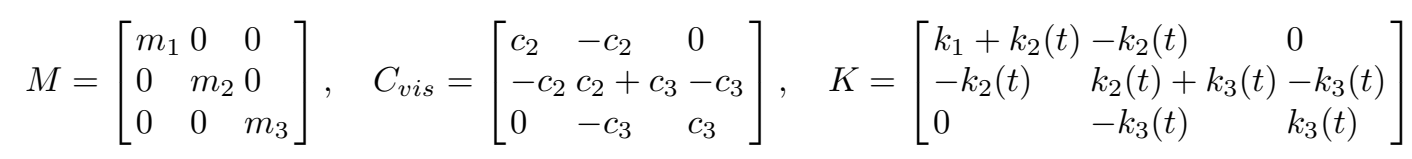

The system is randomly excited by a normal distribution with zero mean and unitary variance. The output vectors in Eq. (1) are corrupted by $1 \%$ (of original signal RMS) white noise. For the simulations, the following numerical values have been adopted [1]:

$\mathrm{m}_{1}=0.5 \mathrm{~kg}, \mathrm{~m}_{2}=1.5 \mathrm{~kg}, \mathrm{~m}_{3}=1 \mathrm{~kg}$

$\mathrm{c}_{2}=0.5 \mathrm{Ns} / \mathrm{m}, \mathrm{c}_{3}=0.3 \mathrm{Ns} / \mathrm{m}$

$\mathrm{k}_{1}=300 \mathrm{~N} / \mathrm{m}, \mathrm{k}_{20}=100 \mathrm{~N} / \mathrm{m}, \mathrm{k}_{21}=60 \mathrm{~N} / \mathrm{m}, \mathrm{k}_{22}=20 \mathrm{~N} / \mathrm{m}, \mathrm{k}_{30}=120 \mathrm{~N} / \mathrm{m}, \mathrm{k}_{31}=72 \mathrm{~N} / \mathrm{m}, \mathrm{k}_{32}=24 \mathrm{~N} / \mathrm{m}$

$\mathrm{p}_{21}=170 \mathrm{~s}, \mathrm{p}_{22}=85 \mathrm{~s}, \mathrm{p}_{31}=141.67 \mathrm{~s}, \mathrm{p}_{32}=94.44 \mathrm{~s}$

In Fig. 2 the presented methods are applied to the numerical system in order to examine the second natural frequency, which is the most varying frequency.

In Fig. 2. A it can be noted that the curve obtained by the estimation is very close to the theoretical one and this confirms that the extension of the LTI methods to the LTV systems is very satisfying. In Fig. 2.B the estimated curve is compared with the predictive one, with $d=5 \mathrm{~s}, p_{r}=5 \mathrm{~s}$. Although there are some little differences in the higher part of the frequency, the trend of the prediction reproduces quite well the estimated one.

\section{Experimental example: A model of railway bridge}

A scaled model of a railway bridge, located in the laboratory of the Department of Mechanics of the Politecnico di Torino, is shown in Fig. 3. It is composed by a metallic plate and a pair of rails, in order to allow the passage of a carriage, which can contain some weights. 


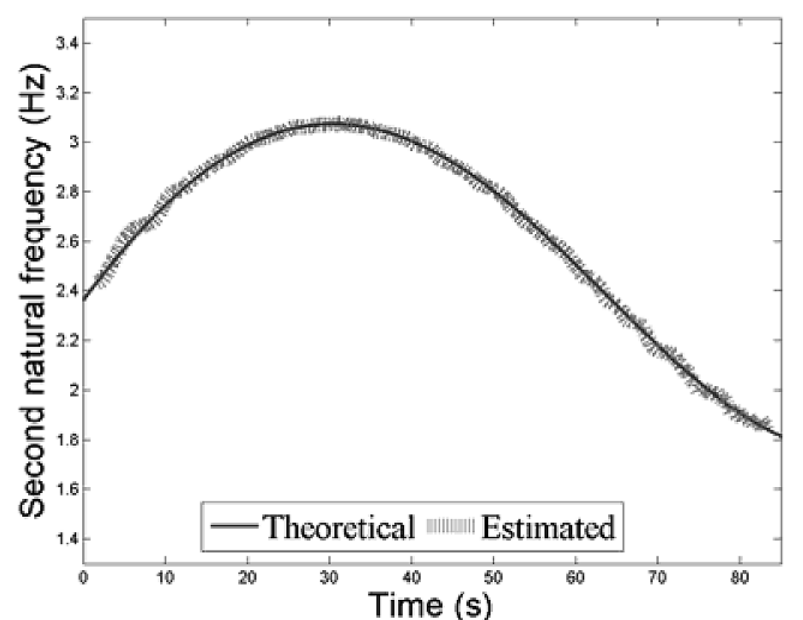

A

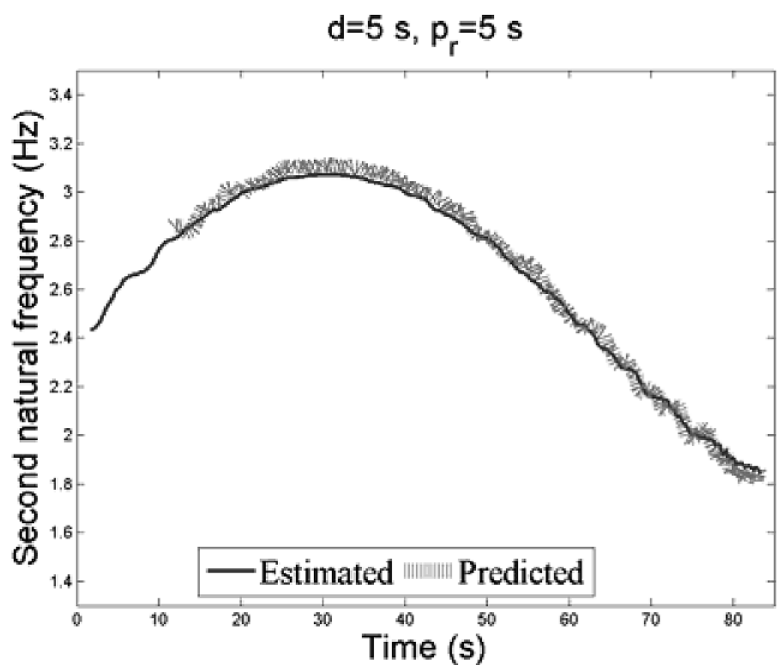

$\mathrm{B}$

Fig. 2. Second natural frequency. A: Comparison theoretical-estimated. B: Comparison estimated-predicted.

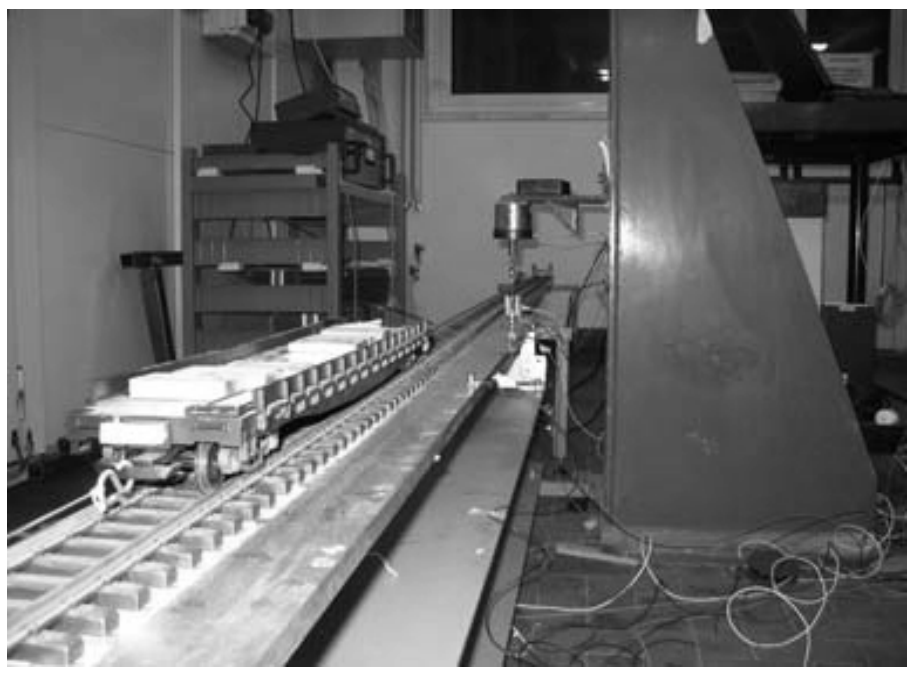

Fig. 3. The model of railway bridge.

A shaker was used to generate the input signal, while an electric motor generated the motion of the carriage over the plate at constant speed. Ten sensors were placed along the plate in order to register the accelerations of the structure.

Two types of experiments were conducted, varying the weights over the train: in the first case a mass $m_{1}=4.4 \mathrm{~kg}$ was chosen, while in the second case a mass $m_{2}=1.2 \mathrm{~kg}$ was selected.

The results of the estimation method for both the situations are depicted in Fig. 4.

Figure 4 shows that the variation of the first natural frequency for the case with mass $m_{1}$ is obviously larger than respect to the case with mass $m_{2}$. In the following only the first case will be considered.

In Fig. 5 a comparison between the results of the estimation method and the predictive method is done on the first two natural frequencies of the bridge.

Both Fig. 5.A and 5.B show that the prediction is able to follow and reproduce every variation in the natural frequencies. However, different values of $d$ and $p_{r}$ should be selected with particular care, depending on the natural frequency to be analyzed. 


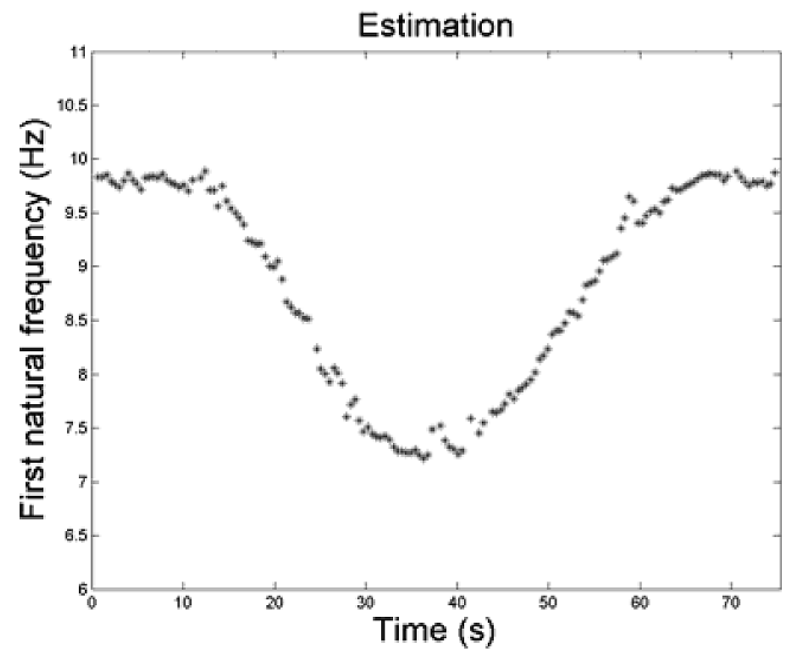

A

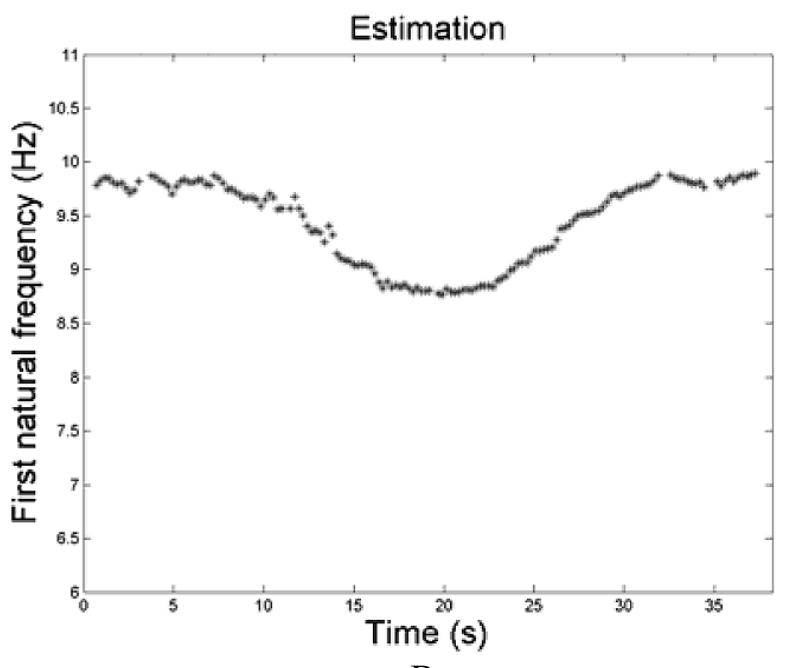

B

Fig. 4. Estimation of the first natural frequency. A: mass $m_{1}$. B: mass $m_{2}$.

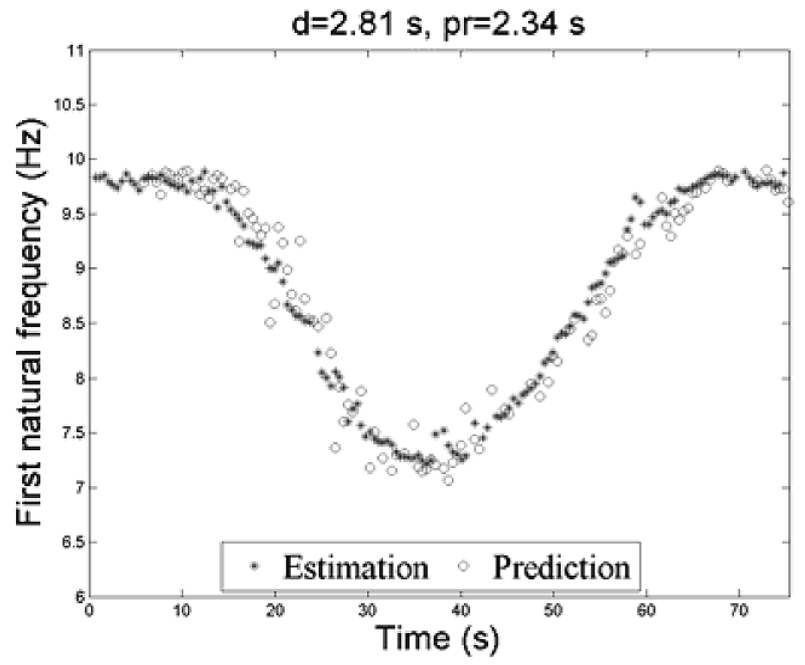

A

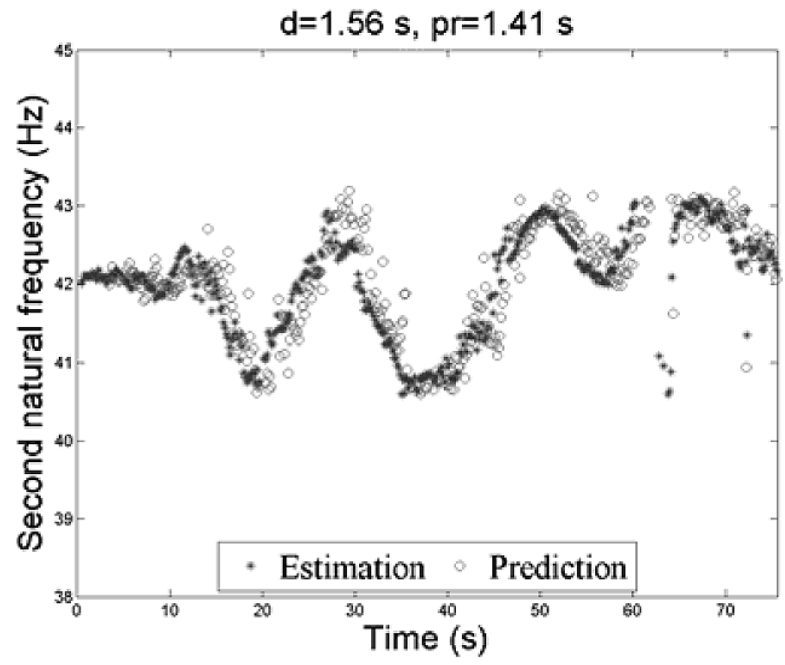

B

Fig. 5. Comparison between estimation and prediction. A: first frequency. B: second frequency.

\section{Conclusions}

An estimation method and a predictive method for the modal parameters have been presented. The first is a direct extension of the subspace methods for LTI systems and it obtains good results in identifying both a numerical and an experimental LTV system. The predictive method has been investigated through its mathematical aspects and through the possible applications. The main idea is that in LTV systems there is a change of the subspace generated by the columns of the observability matrix at two successive instants, and this fact can be used to predict the subspace at a future instant.

It has been demonstrated that the method is able to reproduce the trend of the real or estimated frequencies, and so it can be considered reliable in predicting the behaviour of a LTV system in the near future. 


\section{Acknowledgements}

This work is supported by the Italian MIUR (Ministero dell'Istruzione, dell'Universit à e della Ricerca) in the framework of PRIN (Progetti di Rilevante Interesse Nazionale) 2007 Research Program, named "Monitoring and diagnostics of railway bridges by means of the analysis of the dynamic response due to train crossing"

\section{References}

[1] A.G. Poulimenos and S.D. Fassois, Parametric Time-Domain Methods for Non-Stationary Random Vibration Modelling and Analysis - A Critical Survey and Comparison, Mechanical Systems and Signal Processing 20 (2006), 763-816.

[2] P. Van Overschee and B. De Moor, Subspace Identification for Linear Systems: Theory, Implementation, Applications, Kluwer Academic Publishers Boston/London/Dordrecht, 1996.

[3] L. Garibaldi, S. Marchesiello and E. Bonisoli, Identification and up-dating over the Z24 benchmark, Mechanical Systems and Signal Processing 17 (2003), 153-161.

[4] K. Liu, Identification of Linear Time-Varying Systems, Journal of Sound and Vibration 206 (1997), 487-505

[5] K. Liu, Extension of Modal Analysis to Linear Time-Varying Systems, Journal of Sound and Vibration 226 (1999), $149-167$.

[6] Z.Y. Shi, S.S. Law and H.N. Li, Subspace-Based Identification of Linear Time-Varying System, AIAA Journal 45 (2007), $2042-2050$.

[7] K. Liu and L. Deng, Experimental verification of an algorithm for identification of linear time-varying systems, Journal of Sound and Vibration 279 (2005), 1170-1180.

[8] K. Liu and L. Deng, Identification of pseudo-natural frequencies of an axially moving cantilever beam using a subspace-based algorithm, Mechanical Systems and Signal Processing 20, 94-113.

[9] K. Kameyama and A. Ohsumi, Subspace-Based Prediction of Linear Varying Stochastic Systems, Automatica 43 (2007), $2009-2021$.

[10] W. Favoreel, B. De Moor and M. Gevers, SPC: Subspace Predictive Control, Proceedings of the 14th IFAC World Congress, Beijing, 199, $235-240$. 

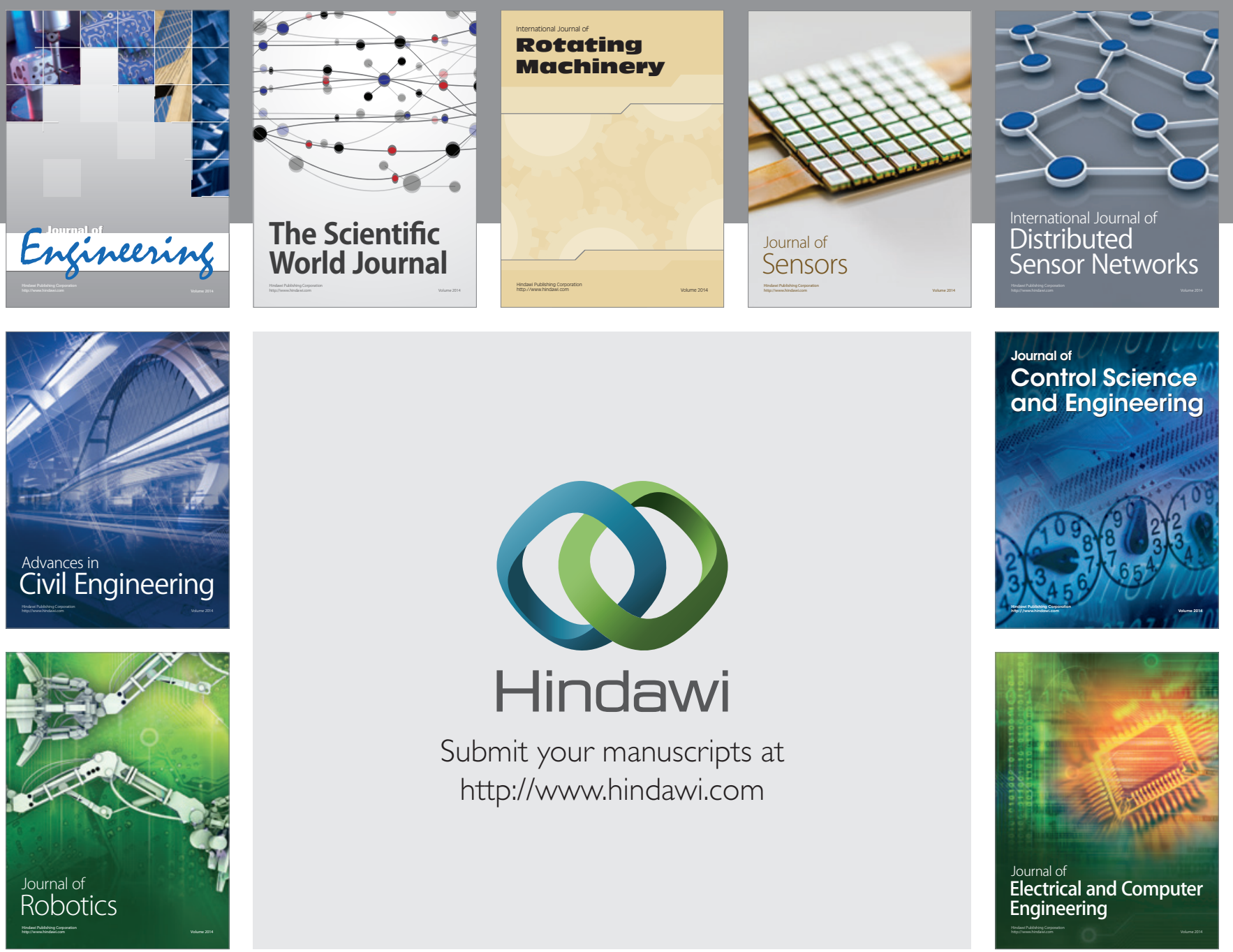

Submit your manuscripts at

http://www.hindawi.com
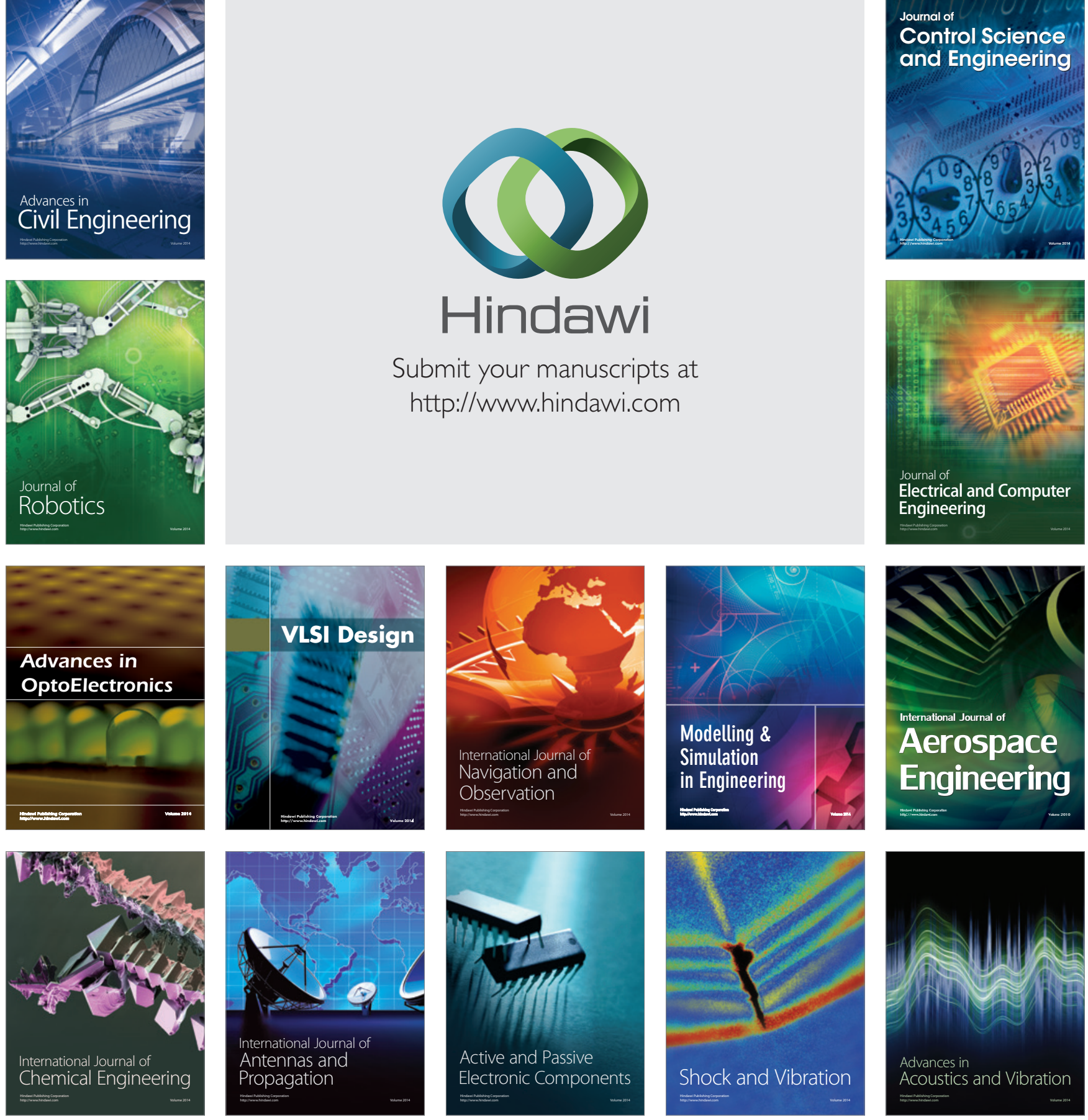